\title{
Optimización de un sistema de biorremediación de hidrocarburos a escala de laboratorio
}

\section{Optimization of a Hydrocarbon Bioremediation System at Laboratory Scale}

\author{
Acuña A.J. \\ Centro de Estudios e Investigación en Microbiología Aplicada \\ (CEIMA) \\ Facultad de Ciencias Naturales \\ Universidad Nacional de la Patagonia San Juan Bosco, Argentina \\ Correo:ajcuna@unpata.edu.ar \\ Tonín N.L. \\ Centro de Estudios e Investigación en Microbiología Aplicada \\ (CEIMA) \\ Facultad de Ciencias Naturales \\ Universidad Nacional de la Patagonia San Juan Bosco, Argentina \\ Correo:ntonin@unpata.edu.ar \\ Pucci O.H. \\ Centro de Estudios e Investigación en Microbiología Aplicada \\ (CEIMA) \\ Facultad de Ciencias Naturales \\ Díaz V. \\ Centro de Estudios e Investigación en Microbiología Aplicada \\ (CEIMA) \\ Facultad de Ciencias Naturales \\ Universidad Nacional de la Patagonia San Juan Bosco, Argentina \\ Correo:victordj_2003@hotmail.com \\ Pucci G.N. \\ Centro de Estudios e Investigación en Microbiología Aplicada \\ (CEIMA) \\ Facultad de Ciencias Naturales \\ Universidad Nacional de la Patagonia San Juan Bosco, Argentina \\ Correo:granapu@unpata.edu.ar \\ Universidad Nacional de la Patagonia San Juan Bosco, Argentina \\ Correo:ceima@unpata.edu.ar
}

Información del artículo: recibido: diciembre de 2010, aceptado: febrero de 2011

\section{Resumen}

El objeto de este trabajo fue optimizar los parámetros de humedad, temperatura y relación de nutrientes para estimar la posibilidad de aplicar la técnica de biorremediación en un suelo contaminado con hidrocarburos. Para esto se realizó una caracterización inicial del suelo contaminado en función de sus características físicas y químicas, su cantidad de microorganismos heterótrofos y degradadores de hidrocarburos. También se estudió la concentración del contaminante por método gravimétrico y por cromatografía gaseosa. Para optimizar humedad y temperatura se trabajó en microcosmos con humedades de $3 \%, 10 \%, 15 \%$ y $20 \%$ y temperaturas de $5^{\circ} \mathrm{C}, 15^{\circ} \mathrm{C}, 28^{\circ} \mathrm{C}$ y $37^{\circ} \mathrm{C}$,

\section{Descriptores}

- biodegradación

- hidrocarburos

- suelo

- Patagonia 
realizando un seguimiento de la mineralización de los hidrocarburos midiendo el $\mathrm{CO}_{2}$ producido. Para optimizar la relación de nutrientes se diseñaron microcosmos diferentes que fueron monitoreados por consumo de oxígeno y por determinación de hidrocarburos por cromatografía gaseosa. Las relaciones C:N:P estudiadas fueron 100:20:2, 100:10:1, 100:5:0,5 y 100:1:0,1. Los resultados obtenidos indican que la mineralización de hidrocarburos fue óptima para humedades de $10 \%$ a $20 \%$ y a temperaturas de $25^{\circ} \mathrm{C}$ a $37^{\circ} \mathrm{C}$ con valores de producción de $\mathrm{CO}_{2}$ de 3000 a $4500 \mathrm{mgCO}_{2} \mathrm{~kg}^{-1}$. La relación óptima C:N:P fue de 100:1:0,1 en la que se observó el mayor consumo de oxígeno y la remoción de $83 \%$ de los hidrocarburos totales determinados por cromatografía gaseosa, con $78 \%$ y $89 \%$ de eliminación de los hidrocarburos n-alcanos y poliaromáticos, respectivamente.

\begin{abstract}
The aim of this study was to optimize the parameters of moisture, temperature and ratio of nutrients to estimate the possibility of applying the technique of bioremediation in a soil contaminated with hydrocarbons. For this, an initial characterization of contaminated soil was made according to their physical and chemical characteristics and the number of heterotrophic and hydrocarbon degraders bacteria. Also the contaminant concentration by gravimetric method and by gas chromatography was studied. To optimize moisture and temperature, microcosms with moisture of $3 \%$, $10 \%, 15 \%$ and $20 \%$ and temperatures of $5^{\circ} \mathrm{C}, 15^{\circ} \mathrm{C}, 28^{\circ} \mathrm{C}$ and $37^{\circ} \mathrm{C}$ were used. The monitoring of the mineralization of hydrocarbons was performed by measuring the $\mathrm{CO}_{2}$ produced. To optimize the ratio of nutrients, different microcosms were designed and were monitored by oxygen consumption and by determination of hydrocarbons by gas chromatography. The C:N:P relationships studied were 100:20:2, 100:10:1, 100:5:0,5 and 100:1:0,1. The results indicate that the mineralization of hydrocarbons was optimal for moisture of $10 \%$ to $20 \%$ and temperatures of $25^{\circ} \mathrm{C}$ to $37^{\circ} \mathrm{C}$ with $\mathrm{CO}_{2}$ production values of 3000-4500 $\mathrm{mgCO}_{2} \mathrm{~kg}^{-}$. The optimal C:N:P ratio was 100:1:0,1 in which the highest oxygen consumption was and the elimination of $83 \%$ of total hydrocarbons determined by gas chromatography with $78 \%$ and $89 \%$ of $n$-alkanes and polyaromatic hydrocarbons elimination, respectively.
\end{abstract}

\section{Introducción}

La Patagonia es una zona semiárida que sufre constantemente erosión eólica y bajas precipitaciones, presentando un suelo con bajo contenido de humedad. A su vez, su franja costera está expuesta a una explotación petrolera intensa, con lo cual se producen contaminaciones de diferentes magnitudes, entre las que se encuentran los derrames de hidrocarburos en suelo. Diferentes son las tecnologías aplicables para recuperar suelos contaminados con hidrocarburos. Los métodos biológicos demostraron ser eficientes y adecuados (Maila y Cloete, 2004; Johnsena et al., 2005), debido a que causan menor impacto en el sitio del problema. Las técnicas clásicas de biorremediación, término que define al proceso mediante el cual los microorganismos presentes en un sitio producen la eliminación de un contaminante, son bioaumento y bioestimulación, am- bas con la posibilidad de ser aplicadas in situ o ex situ (Van Hamme et al., 2003). Llamamos bioaumento a la incorporación de microorganismos especializados al sitio contaminado con el fin de mejorar el rendimiento del proceso de biorremediación. La bioestimulación consiste en estimular a los microorganismos de un ambiente natural por medio del agregado de nutrientes, para así mejorar la eliminación de los contaminantes. Es común la incorporación de nitrógeno, fósforo, potasio y humedad en el suelo contaminado para adecuar la relación carbono:nitrógeno:fósforo ( $\mathrm{C}: \mathrm{N}: \mathrm{P}$ ) del mismo (Atlas, 1995). Para que dicho proceso pueda llevarse a cabo, hay una serie de factores que deben conocerse. Estos factores son la composición química del contaminante, las características del hábitat y de los microorganismos donde se encuentra el contaminante (Alvarez e Illman, 2006). Tanto la humedad y temperatura como la relación de nutrientes en el suelo, son datos de gran im- 
portancia a tener en cuenta debido a que son responsables de permitir un buen desarrollo de microorganismos (Leahy y Colwell, 1990), logrando que el número de ellos sea suficiente en la matriz del suelo para que el proceso de biorremediación de hidrocarburos sea eficiente.

El objetivo de este trabajo fue optimizar a escala de laboratorio, un sistema de biorremediación de hidrocarburos en un suelo contaminado estudiando los parámetros de humedad, temperatura y relación de nutrientes.

\section{Metodología}

Muestra de suelo

Se estudió un suelo contaminado con residuos de la industria petrolera provenientes del noreste de la ciudad de Comodoro Rivadavia, Argentina. La muestra se obtuvo de la toma de 25 submuestras de aproximadamente un kilogramo a partir de un área de $100 \mathrm{~m}^{2}$ a una profundidad comprendida entre 10 y $30 \mathrm{~cm}$ (Atlas y Bartha, 2002). Éstas fueron mezcladas, pasadas por un tamiz de $2 \mathrm{~mm}$ de abertura para eliminar todas las piedras existentes, y por cuarteos sucesivos se tomó una porción de $2 \mathrm{~kg}$ sobre la cual se realizaron las determinaciones.

\section{Estudio inicial del suelo}

Análisis físico y químico

Las determinaciones de humedad, materia orgánica, densidad real, densidad aparente, porosidad y de capacidad de retención de agua (CRA) se realizaron según lo propuesto por García-Trejo en 1981. El pH, cationes y aniones se analizaron sobre un extracto de suelo 1:2,5 en agua destilada. La determinación de $\mathrm{pH}$ fue realizada por potenciometría con electrodo de vidrio y el contenido de carbonato y bicarbonato por titulación con ácido clorhídrico $0,1 \mathrm{~N}$.

El calcio y magnesio se efectuaron por complejometría con EDTA, a pH 12, para el primero de ellos, utilizando murexida como indicador y a $\mathrm{pH} 10$ con negro de ericromo T como indicador, para el segundo. El cloruro fue determinado por el método de Mohr y el sulfato por su precipitación en medio ácido. El nitrito se determinó colorimétricamente con ácido sulfanílico y 1-naftilamina y el nitrato con brucina en presencia de ácido sulfúrico.

El ión amonio como azul de indofenol y el fosfato con azul de molibdeno (Acuña et al., 2008). La textura del suelo se realizó sobre $25 \mathrm{~g}$ de peso seco utilizando el método del tacto según lo propuesto por Yolcubal et al. (2004). Este método se complementó determinando la composición granulométrica de los mismos, sobre 100 g, utilizando diferentes tamices. Se utilizó un tamiz núm. 40 , con una abertura de $0,42 \mathrm{~mm}$, para conocer la cantidad de arena de tamaño medio, otro núm. 120, con abertura de 0,125 $\mathrm{mm}$, para las partículas de arena fina y otro núm. 170, con abertura de 0,088 mm, para las de arena muy fina. Las partículas que no fueron retenidas por el tamiz núm. 170, se clasificaron como la sumatoria de limo y arcilla.

\section{Análisis microbiológico}

Los conteos de bacterias heterótrofas y degradadoras de hidrocarburos $(\mathrm{BDH})$ se realizaron por conteo en placa en agra R2A (Reasoner y Geldreich, 1985) y MMPGO (Acuña et al., 2008), respectivamente.

\section{Análisis de hidrocarburos}

El contenido de hidrocarburos totales (HT) se determinó sobre $50 \mathrm{~g}$ de suelo mediante extracción con Soxhlet durante $24 \mathrm{~h}$ con tricloroetano como solvente de extracción. Los hidrocarburos extraídos fueron cuantificados gravimétricamente (Pucci y Pucci, 2003). Las fracciones alifática, aromática y polar de los HT obtenidos fueron separadas por cromatografía en columna de silicagel (Kieselgel 60, 35-70 mesh, Merck). Como solventes de elución se utilizaron hexano, benceno y cloroformometanol 1:1 para los hidrocarburos alifáticos, aromáticos y polares, respectivamente (Speight, 1991). Las fracciones obtenidas se cuantificaron gravimétricamente (Pucci y Pucci 2003).

La fracción de hidrocarburos n-alcanos de C10 a C26 y poliaromáticos se realizó por cromatografía gaseosa. Para esto se tomó un gramo de suelo y se realizó una extracción con $10 \mathrm{~mL}$ de hexano por agitación durante una hora.

La identificación y cuantificación se realizó en un cromatógrafo de gases Varian CP-3800 equipado con una columna capilar (VF5ms) de sílicagel fundida de 30 $\mathrm{m}$ por $0,25 \mathrm{~mm}$ por $0,25 \mu \mathrm{m}$ y un programa de temperatura de $45^{\circ} \mathrm{C}$ iniciales por tres minutos, seguido de una rampa de $45^{\circ} \mathrm{C}$ a $275^{\circ} \mathrm{C}$ a $12^{\circ} \mathrm{C} \cdot \mathrm{min}^{-1}$, finalizando con 12 $\min$ a $275^{\circ} \mathrm{C}$, con una temperatura del inyector y el detector de $200^{\circ} \mathrm{C}$ y $300^{\circ} \mathrm{C}$, respectivamente, y un detector por ionización de llama. Para la identificación y cuantificación de los hidrocarburos se utilizaron los testigos propuestos por la Environmental Protection Agency en sus normas 8015D y 610. 


\section{Optimización del proceso de biodegradación}

Humedad y temperatura

Se diseñaron 16 microcosmos por triplicado en botellas de vidrio de un litro con $100 \mathrm{~g}$ de suelo. Los valores de humedad estudiados fueron $3 \%, 10 \%, 15 \%$ y $20 \%$. Los valores de temperatura ensayados fueron $5^{\circ} \mathrm{C}, 15^{\circ} \mathrm{C}$, $28^{\circ} \mathrm{C}$ y $37^{\circ} \mathrm{C}$. La relación C:N:P de cada microcosmo fue corregida según lo publicado por otros autores a 100:2:0,2 utilizando $\mathrm{K}_{2} \mathrm{HPO}_{3}$ y $\left(\mathrm{NH}_{4}\right)_{2} \mathrm{SO}_{4}$ (Acuña et al., 2008). Los sistemas se incubaron 60 días en oscuridad y se midió dos veces por semana la mineralización de los hidrocarburos presentes determinando el dióxido de carbono $\left(\mathrm{CO}_{2}\right)$ producido en los microcosmos. $\mathrm{El} \mathrm{CO}_{2}$ liberado se fijó en un recipiente dentro del sistema con $3 \mathrm{~mL}$ de $\mathrm{NaOH}$ y el carbonato formado se tituló con ácido clorhídrico 0,1 N.

\section{Nutrientes}

Se utilizaron cuatro microcosmos con $25 \mathrm{~g}$ de suelo en sistemas de $250 \mathrm{~mL}$ de capacidad. La proporción C:N:P utilizada para el sistema 1, 2, 3 y 4 fue de 100:20:2, 100:10:1, 100:5:0,5 y 100:1:0,1, respectivamente. El seguimiento se realizó determinando el consumo de oxígeno utilizando cabezales OxiTop ${ }^{\circledR}$. Al finalizar la experiencia, se realizó la determinación de hidrocarburos en cada microcosmo por cromatografía gaseosa según lo explicado anteriormente. Los microcosmos se incubaron a $28^{\circ} \mathrm{C}$ durante 30 días en oscuridad.

\section{Análisis de datos}

Los resultados obtenidos fueron analizados utilizando análisis de la varianza (ANOVA) con 3 réplicas por nivel. Para estos estudios se utilizó el programa BIOM (Applied Biostatistics Inc., 3 Heritage, Setauket, NY 117II USA) con un nivel de significancia de 5\%. Los resultados mostrados en gráficos y tablas fueron corregidos por gramo de suelo seco, y se expresa el valor medio de los triplicados.

\section{Resultados y discusión}

Estudio inicial del suelo

Para que un proceso de biodegradación de hidrocarburos sea eficiente en un suelo, es necesario conocer cómo son las características del hábitat microbiano. Éstas se encuentran definidas por las características físicas y químicas del suelo (Van Hamme et al., 2003). Así, los valores de capacidad de retención de agua, porosidad y textura observados en la muestra estudiada favorecen el crecimiento microbiológico (Leahy y Colwell, 1990). El suelo estudiado posee una textura areno limoso al tacto con un $7 \%$ de partículas finas, limo y arcilla, y con 93\% de arena, conformada principalmente por arena fina y muy fina. En esta textura se encuentran poros grandes, conformados por las partículas de arena, que permiten la buena aireación del suelo, favoreciendo el desarrollo bacteriano (Voroney, 2007). Asimismo, al estar la arena del suelo conformada por partículas de tamaño fino y muy fino, el tamaño de los poros es tal que favorecería la retención del agua incorporada en el suelo (Yolcubal et al., 2004). Por otro lado, la fracción de limo y arcilla que forma parte de este suelo, le proporciona una fracción que es capaz de retener por largos periodos el agua incorporada (Coleman et al., 2004), lo que brinda a los microorganismos una humedad adecuada para el desarrollo de su metabolismo por periodos de tiempo más prolongados. La baja concentración de nutrientes biodisponibles (nitrato, nitrito, amonio y fosfato) es una situación desfavorable para el proceso de biorremediación que, de acuerdo a lo expresado por Van-Hamme et al. (2003), podría ser solucionado con un adecuado sistema de bioestimulación.

El número de $\mathrm{BDH}$ encontrado en el suelo fue del mismo orden que para bacterias heterótrofas, demostrando que en el mismo se encuentra una comunidad bacteriana adaptada para biodegradar hidrocarburos (Peressutti et al., 2003). En estudios previos se pudo determinar que en suelos patagónicos con DBH en el orden de $10^{6}$ a $10^{8}$ se pueden realizar procesos de biodegradación de hidrocarburos en forma óptima (Acuña et al., 2008).

El contenido de hidrocarburos encontrado en el suelo fue $5,4 \%$ (tabla 1). Estos se encontraron constituidos por $35 \%$ de hidrocarburos alifáticos, $14 \%$ de hidrocarburos aromáticos y $51 \%$ de hidrocarburos polares, esto demuestra que en el suelo se encuentra un alto porcentaje de hidrocarburos polares, característico del petróleo de la región. Este grupo de hidrocarburos es el más difícil de ser biodegradado por los microorganismos. Sin embargo, los hidrocarburos alifáticos y aromáticos presentes en el suelo son susceptibles al proceso de biodegradación, siendo la fracción aromática biodegradada de forma más lenta que la fracción alifática (Pucci y Pucci, 2003). Los HT determinados por cromatografía gaseosa (tabla 2) fueron cercanos a 1,5\% con valores de $0,05 \%$ de HPA, siendo mayoritario el contenido de fenantreno, fluorantreno, criseno, antraceno, benzo(a)antraceno y 2-metilnaftaleno. Los n-alcanos identificados se encontraron en una concentración aproximada de 
0,5\% con compuestos de C13 a C20 como su fracción mayoritaria, observándose la mayor concentración para n-heptadecano. Tanto los n-alcanos como los HPA, son compuestos que en sistemas de biodegradación pueden ser metabolizados por los microorganismos presentes (Ghazali et al., 2004, Jacques et al., 2008), siendo los HPA el grupo que presenta la menor velocidad de biodegradación (Straube et al., 2003).

\section{Optimización del proceso de biodegradación}

La mayor mineralización de hidrocarburos se observó entre $25^{\circ} \mathrm{C}$ y $37^{\circ} \mathrm{C}$ con un porcentaje de humedad entre $10 \%$ y $20 \%$, con valores de producción de $\mathrm{CO}_{2}$ de aproximadamente $3000 \mathrm{mgCO} \cdot \mathrm{kg}^{-1}$ a $4500 \mathrm{mgCO} \cdot \mathrm{kg}^{-1}$ (figura 1).

Se observó que a $15^{\circ} \mathrm{C}$, en el rango de $10 \%$ a $20 \%$ de humedad, la producción de $\mathrm{CO}_{2}$ fue de aproximadamente $3000 \mathrm{mgCO}_{2} \cdot \mathrm{kg}^{-1}$. Por otro lado, con $3 \%$ de hu- medad y entre $25^{\circ} \mathrm{C}$ y $37^{\circ} \mathrm{C}$, se comprobó que la mineralización de los hidrocarburos produjo aproximadamente $2000 \mathrm{mgCO} \cdot \mathrm{kg}^{-1}$. Los menores valores de mineralización $\left(1000 \mathrm{mgCO} \cdot \mathrm{kg}^{-1}\right)$ se observaron a $5^{\circ} \mathrm{C}$ y en todo el rango de humedad estudiado, y a $15^{\circ} \mathrm{C}$ con una humedad comprendida entre $3 \%$ y $5 \%$. La bibliografía cita diversas proporciones de nutrientes como las más efectivas para un proceso de biodegradación en suelos (Van Hamme et al., 2003), pero estas proporciones varían ampliamente y las distintas cantidades influyen significativamente en el costo del proceso, no sólo por el precio, sino por su influencia en la velocidad de degradación, lo que implicaría un mayor tiempo de proceso (Chaîneau et al., 2005). A medida que aumentó la concentración de nutrientes en el suelo, disminuyó el consumo de oxígeno, siendo $48124 \mathrm{mgO}_{2} \cdot \mathrm{kg}^{-1}$ para el sistema 4, $30077 \mathrm{mgO}_{2} \cdot \mathrm{kg}^{-1}$ para el sistema 3 y 25878 $\mathrm{mgO}_{2} \cdot \mathrm{kg}^{-1}$ y $12712 \mathrm{mgO}_{2} \cdot \mathrm{kg}^{-1}$ para los sistemas 2 y 1 , respectivamente (figura 2).

\begin{tabular}{|c|c|c|c|c|}
\hline \multicolumn{3}{|c|}{ Análisis físico } & \multicolumn{2}{|c|}{ Análisis químico $\left(\mathrm{mg} \cdot \mathrm{kg}^{-1}\right)$} \\
\hline \multicolumn{2}{|l|}{$\mathrm{pH}$} & 8,0 & Cloruro & 1,6 \\
\hline \multicolumn{2}{|c|}{ Humedad (\%) } & 6,1 & Sulfato & 385 \\
\hline \multicolumn{2}{|c|}{ Materia orgánica (\%) } & 5,2 & Carbonato & $\mathrm{Nd}$ \\
\hline \multicolumn{2}{|c|}{ Densidad aparente (g. $\left.\mathrm{cm}^{3-1}\right)$} & 1,1 & Bicarbonato & 0,4 \\
\hline \multicolumn{2}{|c|}{ Densidad real (g.cm $\left.{ }^{3-1}\right)$} & 2,2 & Calcio & $\mathrm{Nd}$ \\
\hline \multicolumn{2}{|c|}{ Porosidad $(\%)$} & 57 & Magnesio & $\mathrm{Nd}$ \\
\hline \multirow{2}{*}{\multicolumn{2}{|c|}{ Capacidad retención de agua (\%) }} & 47 & Nitrito & 4,9 \\
\hline & & & Nitrato & 21,5 \\
\hline \multicolumn{2}{|c|}{ Análisis de Textura (\%) } & & Amonio & 0,4 \\
\hline Tacto & \multicolumn{2}{|r|}{ Areno Limoso } & Fosfato & 1,7 \\
\hline Arena me & & 4 & \multicolumn{2}{|c|}{ Análisis de hidrocarburos $\left(\mathrm{mg} \cdot \mathrm{kg}^{-1}\right)$} \\
\hline Arena fin & \multicolumn{2}{|r|}{$\begin{array}{c}4 \\
61\end{array}$} & Totales & 54200 \\
\hline Arena muy & & 28 & Alifáticos & 18970 \\
\hline \multirow{2}{*}{\multicolumn{2}{|c|}{ Limo y arcilla }} & \multirow[t]{2}{*}{7} & Aromáticos & 7588 \\
\hline & & & Polares & 27642 \\
\hline Compuesto & $\mathrm{mg} \cdot \mathrm{kg}^{-1}$ & & Compuesto & $\mathrm{mg} \cdot \mathrm{kg}^{-1}$ \\
\hline n-Decano & 17,398 & & Naftaleno & 11,529 \\
\hline n-Undecano & 66,477 & & Metilnaftaleno & 41,620 \\
\hline n-Dodecano & 173,314 & & Metilnaftaleno & 5,079 \\
\hline n-Tridecano & 444,649 & & Acenaftileno & 19,957 \\
\hline n-Tetradecano & 427,728 & & Acenaftaleno & 4,259 \\
\hline n-Pentadecano & 600,986 & & Fluoreno & 11,675 \\
\hline n-Hexadecano & 476,200 & & Fenantreno & 109,144 \\
\hline n-Heptadecano & 994,502 & & Antraceno & 76,482 \\
\hline n-Octadecano & 258,892 & & Fluorantreno & 90,788 \\
\hline n-Nonadecano & 556,405 & & Pireno & 23,343 \\
\hline n-Eicosano & 444,860 & Benz & zzo(a)antraceno & 75,252 \\
\hline n-Eneicasano & 190,184 & & Criseno & 78,881 \\
\hline n-Docosano & 168,094 & Benzo & o(b)fluorantreno & 7,878 \\
\hline n-Tricosano & 217,919 & Benzo & $\mathrm{o}(\mathrm{k})$ fluorantreno & 15,220 \\
\hline n-Tetracosano & 168,126 & & enzo(a)pireno & 15,824 \\
\hline n-Pentacosano & 111,735 & & & \\
\hline n-Hexacosano & 141,067 & & HC Totales & 14104,364 \\
\hline & & $\mathrm{n}-\mathrm{A}$ & Alcanos Totales & 5458,533 \\
\hline & & & HPA Totales & 586,931 \\
\hline
\end{tabular}

Tabla 1. Características físicas y químicas y concentración de hidrocarburos de la muestra de suelo utilizada en el estudio

Tabla 2. Concentración de hidrocarburos inicial por cromatografía gaseosa en la muestra de suelo utilizada en el estudio 


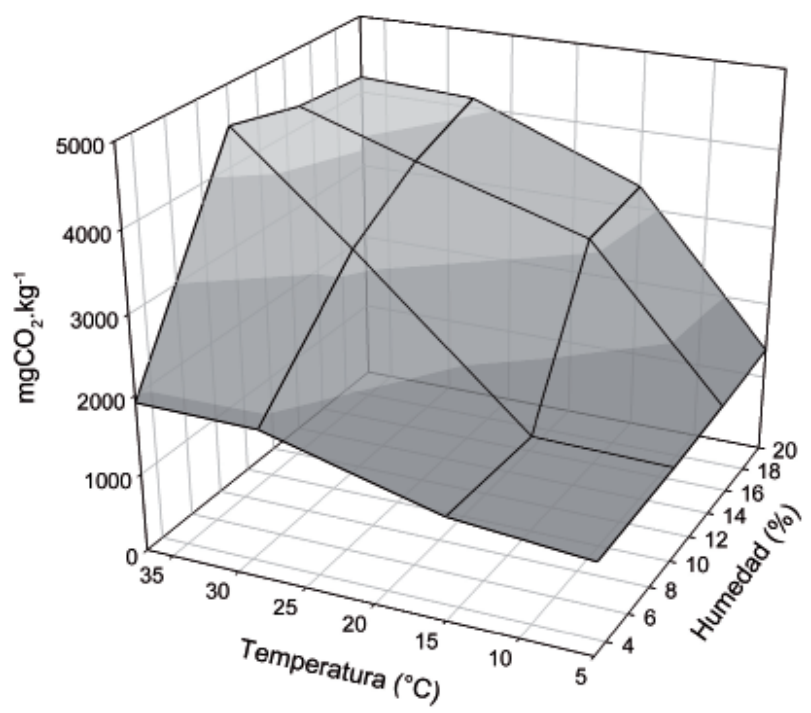

Figura 1. Cantidad de dióxido de carbono producido en los microcosmos utilizados en la experiencia de optimización de humedad y temperatura

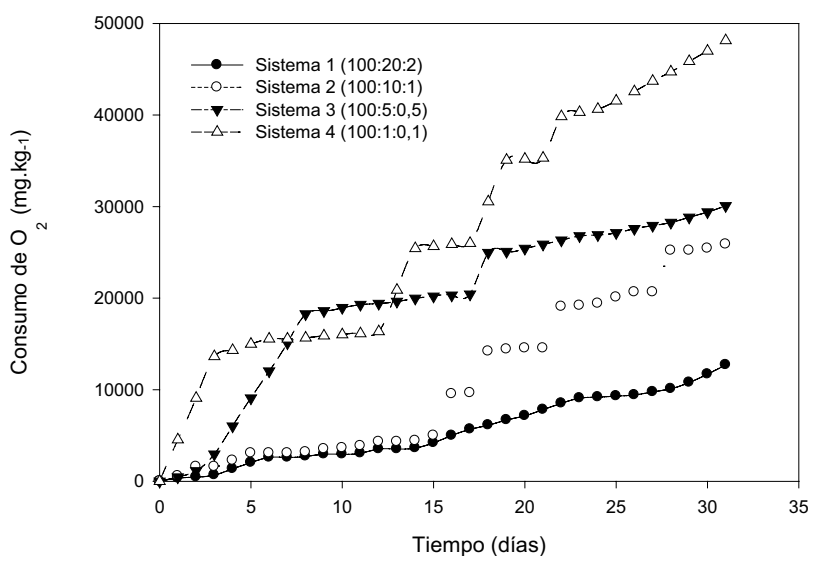

Figura 2. Seguimiento del consumo de oxígeno en los microcosmos utilizados en la experiencia de optimización de nutrientes

El aumento de nutrientes en el suelo también produjo un alargamiento de la fase de latencia de la comunidad bacteriana presente, capaz de biodegradar hidrocarburos. Con respecto a los hidrocarburos totales, en el sistema 1 se observó un porcentaje de biodegradación de $9 \%$, mientras que en los sistemas 2 y 3, este valor fue $29 \%$ para ambos. En el sistema 4, con una relación $\mathrm{C}: \mathrm{N}: \mathrm{P}$ de 100:1:0,1, la biodegradación de los hidrocarburos totales fue $83 \%$ (tabla 3). En este sistema se pudo observar que los hidrocarburos n-alcanos se eliminaron en $78 \%$ y que los HPA en $89 \%$ con una buena biodegradación para todos los compuestos determinados. Los suelos de la Patagonia, Argentina, tienen un marcado déficit de nitrógeno y de fósforo y las comunidades bac- terianas presentes en estos sitios se encuentran altamente adaptadas a estas condiciones ambientales (Mazzarino et al., 1998). La incorporación de nutrientes en altas concentraciones puede provocar un efecto adverso al deseado, disminuyendo el número de bacterias degradadoras de hidrocarburos o su actividad y por consiguiente, la biodegradación (Chaîneau et al., 2005). En los sistemas 2 y 3 , se encontraron valores similares de biodegradación entre $27 \%$ y $22 \%$ para los n-alcanos

Tabla 3. Concentración de hidrocarburos final por cromatografía gaseosa en el suelo de los microcosmos utilizados en la experiencia de optimización de nutrientes

\begin{tabular}{|c|c|c|c|c|}
\hline Compuesto & Sistema 1 & Sistema 2 & Sistema 3 & Sistema 4 \\
\hline n-Decano & 0,000 & 0,000 & 0,000 & 0,000 \\
\hline n-Undecano & 1,569 & 2,228 & 2,173 & 0,000 \\
\hline n-Dodecano & 25,717 & 31,581 & 31,312 & 7,865 \\
\hline $\mathrm{n}$-Tridecano & 193,297 & 198,547 & 183,093 & 40,707 \\
\hline n-Tetradecano & 363,868 & 317,285 & 319,516 & 93,571 \\
\hline n-Pentadecano & 527,438 & 430,589 & 447,275 & 111,850 \\
\hline n-Hexadecano & 470,980 & 398,387 & 421,919 & 116,688 \\
\hline n-Heptadecano & 990,730 & 787,838 & 824,023 & 208,286 \\
\hline n-Octadecano & 256,770 & 214,014 & 245,622 & 107,661 \\
\hline n-Nonadecano & 394,926 & 546,876 & 551,990 & 122,099 \\
\hline n-Eicosano & 441,880 & 435,770 & 132,920 & 95,799 \\
\hline n-Eneicasano & 175,880 & 189,430 & 182,947 & 105,703 \\
\hline n-Docosano & 198,920 & 170,550 & 159,981 & 64,631 \\
\hline n-Tricosano & 220,084 & 186,769 & 161,743 & 55,611 \\
\hline n-Tetracosano & 159,393 & 157,683 & 105,829 & 29,981 \\
\hline n-Pentacosano & 112,109 & 101,455 & 76,677 & 19,763 \\
\hline n-Hexacosano & 69,552 & 89,480 & 48,555 & 8,039 \\
\hline Naftaleno & 0,466 & 0,628 & 0,655 & 4,165 \\
\hline 2-Metilnaftaleno & 2,897 & 2,897 & 3,002 & 1,018 \\
\hline 1-Metilnaftaleno & 3,304 & 3,313 & 3,234 & 1,428 \\
\hline Acenaftileno & 18,990 & 18,328 & 17,062 & 2,505 \\
\hline Acenaftaleno & 1,995 & 1,871 & 1,972 & 0,603 \\
\hline Fluoreno & 12,095 & 10,270 & 10,907 & 4,014 \\
\hline Fenantreno & 110,000 & 67,074 & 84,545 & 8,152 \\
\hline Antraceno & 31,030 & 21,358 & 75,332 & 2,647 \\
\hline Fluorantreno & 89,452 & 13,678 & 14,449 & 7,343 \\
\hline Pireno & 24,200 & 6,156 & 11,669 & 3,940 \\
\hline Benzo(a)antraceno & 76,224 & 42,976 & 30,946 & 1,907 \\
\hline Criseno & 77,224 & 53,267 & 41,427 & 25,380 \\
\hline $\begin{array}{c}\operatorname{Benzo}(b) \\
\text { fluorantreno }\end{array}$ & 1,331 & 6 & 0 & 0,000 \\
\hline $\begin{array}{c}\operatorname{Benzo}(k) \\
\text { fluorantreno }\end{array}$ & 15,181 & 1,922 & 0,000 & 0,000 \\
\hline Benzo(a)pireno & 13,034 & 1,378 & 2,333 & 0,000 \\
\hline HC Totales & 12796,451 & 10028,353 & 9992,755 & 2432,491 \\
\hline n-Alcanos Totales & 4603,113 & 4258,482 & 3895,575 & 1188,255 \\
\hline HPA Totales & 477,422 & 245,853 & 297,533 & 63,103 \\
\hline
\end{tabular}


y $49 \%$ a $58 \%$ para HPA, encontrándose que los n-alcanos se biodegradaron en menor medida a partir de $\mathrm{C} 16$ con excepción de n-eicosano y n-hexacosano, en el sistema 3, que fueron biodegradados en $70 \%$ y $66 \%$, respectivamente. Con respecto a los HPA, la mayoría se biodegradó en un menor porcentaje, observándose, en ambos sistemas, que acenaftileno y fluoreno fueron muy poco biodegradados. En el sistema 1 los n-alcanos totales fueron biodegradados en $16 \%$ con un descenso marcado en la biodegradación a partir de C14, los HPA fueron biodegradados en 19\%, observándose que sólo los hidrocarburos naftaleno, 2-metilnaftaleno, acenaftaleno, fenantreno y benzo(b)fluorantreno fueron biodegradados en un porcentaje mayor a $50 \%$.

\section{Conclusiones}

Los datos presentados permiten establecer que en el suelo estudiado es posible llevar adelante un proceso de biodegradación de hidrocarburos. La base del proceso está garantizada debido a que no se encontraron características físicas, químicas y microbiológicas que impidan que se lleve adelante. El proceso funciona de manera óptima con una relación C:N:P de 100:1:0,1 y a temperaturas de $25^{\circ} \mathrm{C}$ a $37^{\circ} \mathrm{C}$ con humedad de $10 \%$ a $20 \%$, destacándose que a $15^{\circ} \mathrm{C}$ se puede realizar una eficiente biodegradación de los hidrocarburos presentes, manteniendo la humedad del suelo entre los valores mencionados anteriormente.

\section{Referencias}

Acuña A., Pucci O., Pucci G. Caracterización de un proceso de biorremediación de hidrocarburos en deficiencia de nitrógeno en un suelo de la Patagonia Argentina. Ecosistemas, (17):85-93, 2008.

Alvarez P., Illman W. Introduction to Bioremediation, en su: Bioremediation and Natural Attenuation: Process Fundamentals and Mathematical Models, Nueva Jersey, John Wiley \& Sons, Inc, 2006, pp. 1-23.

Atlas R., Bartha R. Los microorganismos en sus hábitat naturales: microbiología del aire, del agua y del suelo, en su: Ecología microbiana y microbiología ambiental, 4a ed., Madrid, Pearson Educación SA, 2002, pp. 329-380.

Atlas R. Bioremediation of petroleum pollutans. Int. Biodeterior. Biodegrd, (1):317-327, 1995.

Chaîneau C., Rougeux G., Yéprémian C. Effects of Nutrient on the Biodegradation of Crude Oil and Associated Microbial Populations in the Soil. Soil Biol. \& Biochem., (37):1490-1497, 2005.

Coleman D., Crossley D., Hendrix P. Historical Overview of Soils and the Fitness of the Soil Environment, en su: Fundamentals of Soil Ecology, Athens, Elsevier, 2004, pp. 1-21.
Environmental Protection Agency. Método 610: Polynuclear Aromatic Hydrocarbons.

Environmental Protection Agency. Método 8015D: Nonhalogenated Organics Using GC/FID.

García-Trejo A. Propiedades fisicoquímicas del suelo, en su: Experimentos en microbiología del suelo, México DF, Compañía Editorial Continental SA, 1981, pp. 14-39.

Ghazali F., Rahman R., Salleh A., Basri M. Biodegradation of Hydrocarbons in Soil by Microbial Consortium. Internat. Biodet. Biodeg., (54):61-67, 2004.

Jacques R., Okeke B., Bento F., Teixeira A., Peralba M., Camargo F. Microbial Consortium Bioaugmentation of a Polycyclic Aromatic Hydrocarbons Contaminated Soil. Bioresource Technol., (99):2637-2643, 2008.

Johnsena A., Wick L., Harmsb H. Principles of Microbial PAHDegradation in Soil. Environ. Pollut., (133):71-84, 2005.

Leahy J, Colwell R. Microbial Degradation of Hydrocarbons in the Environment. Microbiol. Rev., (54):305-315, 1990.

Maila M., Cloete T. Bioremediation of Petroleum Hydrocarbons Through Landfarming: are Simplicity and Cost-Effectiveness the Only Advantages? Rev. Environ. Scien. Biotechnol., (3):349360, 2004.

Mazzarino M., Bertiller M., Sain C., Satti P., Coronato F. Soil Nitrogen Dynamics in Northeastern Patagonia Steppe under Different Precipitation Regimes. Plant and Soil., (202):125-131, 1998.

Peressutti S., Alvarez H., Pucci O. Dynamics of Hydrocarbon-Degrading Bacteriocenosis of an Experimental Oil Pollution in Patagonian Soil. Intern. Biodet. and Biodeg., (52):21-30, 2003.

Pucci G., Pucci O. Biodegradabilidad de componentes de mezclas naturales de hidrocarburos previamente sometidas a landfarming. Rev. Argent. Microbiol., (35):62-68, 2003.

Reasoner D., Geldreich E. A New Medium for the Enumeration and Subculture of Bacteria from Potable Water. Appl. Environ. Microbiol., (49):1-7, 1985.

Speight J. Composition, en su: The Chemistry and Technology of Petroleum, 2a ed., USA, Marcel Dekker, 1991, pp. 209-253.

Straube W., Nestler C., Hansen L., Ringleberg D., Pritchard P., Jones-Meehan J. Remediation of Polyaromatyc Hydrocarbons (PAHs) through Landfarming with Biostimulation and Bioaugmentation. Acta Biotechnol., (23):179-196, 2003.

Van-Hamme J., Singh A., Ward O. Recent Advances in Petroleum Microbiology. Microbiol. Mol. Biol. Rev., (67):503-549, 2003.

Voroney R. The Soil Habitat, en su: Soil Microbiology and Biochemistry, USA, Elsevier, 2007, pp. 25-49.

Yolcubal I., Brusseau M., Artiola J., Wierenga P., Wilson L. Environmental Physical Properties and Processes, en su: Environmental Monitoring and Cheracterization, USA, Elsevier, 2004, pp. 207-239. 


\section{Semblanza de los autores}

Adrián Javier Acuña. Es técnico universitario en química y bioquímico, egresado de la Universidad Nacional de la Patagonia San Juan Bosco, Argentina. Realizó el doctorado en bioquímica en la misma Universidad. Se desempeña como investigador en el Centro de Estudios e Investigación en Microbiología Aplicada y como docente en la Universidad Nacional de la Patagonia San Juan Bosco.

Natalia Tonín. Es licenciada en protección y saneamiento ambiental, egresada de la Universidad Nacional de la Patagonia San Juan Bosco. Se desempeña como investigador en el Centro de Estudios e Investigación en Microbiología Aplicada y como docente en la Universidad Nacional de la Patagonia San Juan Bosco.

Víctor Díaz. Es licenciado en protección y saneamiento ambiental, egresado de la Universidad Nacional de la Patagonia San Juan Bosco. Desempeña tareas técnicas en el Centro de Estudios e Investigación en Microbiología Aplicada de la Universidad Nacional de la Patagonia San Juan Bosco.

Graciela Natalia Pucci. Es técnica universitaria en química y bioquímica, egresada de la Universidad Nacional de la Patagonia San Juan Bosco, Argentina. Realizó el doctorado en bioquímica en la misma Universidad. Se desempeña como investigadora del Centro de Estudios e Investigación en Microbiología Aplicada y como docente en la Universidad Nacional de la Patagonia San Juan Bosco.

Oscar Héctor Pucci. Es licenciado en bioquímica, egresado de la Universidad Nacional del Sur, Argentina. Obtuvo el doctorado por la Universidad de Buenos Aires, Argentina. Se desempeña como director del Centro de Estudios e Investigación en Microbiología Aplicada y como docente en la Universidad Nacional de la Patagonia San Juan Bosco. 